\title{
Long non-coding RNA small nucleolar RNA host gene 1 knockdown suppresses the proliferation, migration and invasion of osteosarcoma cells by regulating microRNA-424-5p/FGF2 in vitro
}

\author{
ZHUOKAI LI $^{1}$, XIAOHE WANG ${ }^{2}$ and SHUOFU LIANG ${ }^{3}$ \\ ${ }^{1}$ Department of Orthopaedics, Shanghai University of Medicine and Health Sciences Affiliated Zhoupu Hospital, \\ Shanghai 201318; ${ }^{2}$ Department of Orthopaedics, Jinan Zhangqiu District Hospital of TCM, Jinan, Shandong 250200; \\ ${ }^{3}$ Department of Orthopaedics, Zibo Zhoucun People's Hospital, Zibo, Shandong 255300, P.R. China
}

Received March 3, 2020; Accepted January 8, 2021

DOI: $10.3892 /$ etm.2021.9756

\begin{abstract}
The aim of the present study was to clarify the effect of long non-coding RNA (lncRNA) small nucleolar RNA host gene 1 (SNHG1) on the proliferation, migration and invasion of osteosarcoma (OS) cells and to explore the potential underlying mechanisms. The expression levels of SNHG1, microRNA (miR)-424-5p and fibroblast growth factor 2 (FGF2) in OS tissues and cells were detected using reverse transcription-quantitative polymerase chain reaction. OS cell proliferation, migration and invasion were analysed by MTT, wound healing and Transwell invasion assays, respectively. The targeting relationships between SNHG1 and miR-424-5p, as well as between miR-424-5p and FGF2, were confirmed using RNA-binding protein immunoprecipitation and/or dual-luciferase reporter gene assays. The results demonstrated that the expression levels of SNHG1 and FGF2 were upregulated, whereas the expression of miR-424-5p was downregulated in OS tissues and cells. The silencing of SNHG1 significantly inhibited the proliferation, migration and invasion of OS cells. Additionally, FGF2 was shown to be a target of miR-424-5p, which in turn, was a target of SNHG1. miR-424-5p silencing and FGF2 overexpression both reversed the suppressive effects of SNHG1 knockdown on the proliferation, migration and invasion of OS cells. Thus, the silencing of
\end{abstract}

Correspondence to: Dr Shuofu Liang, Department of Orthopaedics, Zibo Zhoucun People's Hospital, 999 Hengxing Road, Zhoucun, Zibo, Shandong 255300, P.R. China

E-mail: liangshuofu276@163.com

Abbreviations: FGF2, fibroblast growth factor 2; lncRNA, long noncoding RNA; miRNA, microRNA; RT-qPCR, reverse transcription-quantitative PCR; OS, osteosarcoma; SNHG1, small nucleolar RNA host gene 1

Key words: osteosarcoma, small nucleolar RNA host gene 1, microRNA-424-5p, invasion, migration, proliferation
SNHG1 may inhibit the proliferation, migration and invasion of OS cells by regulating the miR-424-5p/FGF2 axis.

\section{Introduction}

Osteosarcoma (OS), a type of malignant tumour that is common in teenagers with a worldwide incidence of 3.4 per million people per year (1), typically originates from mesenchymal stem cells $(2,3)$. In the past, amputation was primarily utilised to treat OS, but the curative effect of this remains limited (1). At present, various treatments for OS exist, including systemic chemotherapy, targeted drug therapy, immunotherapy and radiotherapy (4). However, many side effects occur from these processes, such as leukopenia and thrombocytopenia and the survival rate of OS patients remains less than $25 \%(5,6)$. Thus, understanding the mechanisms that underlie OS is critical for developing a new treatment strategy.

Long non-coding RNAs (lncRNAs) are RNAs that do not code for a protein but do serve important roles in cellular processes by regulating specific genes (7). Previous studies have shown that many lncRNAs are involved in the pathogenesis of OS, such as taurine upregulated 1 (TUG1), X-inactive specific transcript (XIST), long intergenic non-protein coding RNA 152 and FOXD2 adjacent opposite strand RNA 1 (FOXD2-AS1) (8-11). Zhang et al (8) found that downregulation of lncRNA TUG1 significantly inhibits OS cell proliferation and promotes apoptosis. Li et al (9) reported that XIST inhibition suppresses the proliferation and invasion of OS cells. Zhang et al (11) showed that FOXD2-AS1 downregulation limits the proliferation, migration and invasion of OS cells. The aforementioned lncRNAs serve as oncogenes in OS. Furthermore, lncRNA small nucleolar RNA host gene 1 (SNHG1) has been demonstrated to facilitate the progression of OS (12-14). Jiang et al (13) determined that upregulation of SNHG1 promotes OS cell proliferation and migration and inhibits apoptosis. In agreement with this, Wang et al (14) found that SNHG1 silencing restrains the proliferation, migration and invasion of OS cells. However, the detailed mechanisms of action of SNHG1 on OS still need to be deciphered.

MicroRNAs (miRNAs) are a kind of small endogenous RNA that can influence the post-transcriptional 
regulation of specific genes (15). Increasing attention has been paid to the anti-tumoral roles of miRNAs in OS, such as miRNA (miR)-206 (16), miR-137 (9), and miR-193-3p (10). miR-424-5p is widely considered to be a suppressor in several types of human cancers, such as glioma (17), cholangiocarcinoma (18) and ovarian cancer (19). Notably, the inhibitory effect of miR-424 on the metastasis of OS cells has also been confirmed (20). LncRNAs can act as competitive endogenous RNAs or sponges of miRNAs. SNHG1 has been reported to facilitate the progression of OS by regulating many miRNAs, including miR-101-3p (12), miR-577 (13) and miR-326 (14). However, the regulatory relationship between SNHG1 and miR-424 remains unclear.

In the present study, the influence of SNHG1 inhibition on the viability, migratory ability and invasive ability of OS cells as well as the potential regulatory mechanisms of SNHG1/miR-424-5p/FGF2 were investigated with the goal of developing a new treatment strategy for OS.

\section{Materials and methods}

Sample collection. Between January 2016 and January 2018, 61 pairs of OS tissue samples and adjacent normal tissues were obtained from patients with OS (average age, 18.6 years old) at the ZhouPu Hospital Affiliated to Shanghai University of Medicine \& Health Sciences. These patients did not receive radiotherapy or chemotherapy before the operation. The protocols of this study were reviewed and approved by the Ethical Committee of ZhouPu Hospital Affiliated to Shanghai University of Medicine \& Health Sciences. All participants provided signed informed consent.

Cell grouping and transfection. The OS cell lines Saos-2, MG63, HOS and U2OS, as well as the human osteoblast cell line hFOB1.19 were purchased from Tongpai (Shanghai) Biotechnology Co., Ltd. The cells were cultured in Dulbecco's modified Eagle's medium (DMEM) containing 10\% foetal bovine serum at $5 \% \mathrm{CO}_{2}, 37^{\circ} \mathrm{C}$ and $95 \%$ humidity. Small interfering (si)RNA-negative control (si-NC; 5'-UUCUCCGAACGU GUCACGUTT-3'), siRNA-SNHG1-1 (si-SNHG1-1, 5'-CAG CAGTTGAGGGTTTGCTGTGTAT-3') and si-SNHG-2 (5'-TTCAACAGCTAGGTTGTCCTT-3') were purchased from Sangon Biotech Co.,Ltd. Overexpression vectors pcDNA-FGF2, pcDNA-SNHG1 and empty vector (pcDNA-NC), along with miR-424-5p mimics, miRNA mimics-NC (miR-NC), miR-424-5p inhibitor and inhibitor $\mathrm{NC}$ were all procured from Guangzhou RiboBio Co., Ltd. The aforementioned agents (all, $50 \mathrm{nM})$ were transfected into the cells $\left(6 \times 10^{5}\right.$ cells/well) using a Lipofectamine RNAiMAX kit (Invitrogen; Thermo Fisher Scientific, Inc.) for $48 \mathrm{~h}$ at $37^{\circ} \mathrm{C}$. Following transfection, the cells were harvested to perform the following experiments.

Reverse transcription quantitative polymerase chain reaction (RT-qPCR). TRIzol ${ }^{\circledR}$ reagent (Invitrogen; Thermo Fisher Scientific, Inc.) was used to extract the total RNA from tissues or cell lines. The GoScript reverse transcription system (Promega Corporation) was used to reverse transcribe the extracted RNA into cDNA. qPCR was performed using the SYBR Green PCR Master mix (Takara Biotechnology Co., Ltd.). The reaction conditions were as follows: $95^{\circ} \mathrm{C}$ for $10 \mathrm{~min}$; followed by 40 cycles at $94^{\circ} \mathrm{C}$ for $10 \mathrm{sec}, 60^{\circ} \mathrm{C}$ for $20 \mathrm{sec}$ and $72^{\circ} \mathrm{C}$ for $34 \mathrm{sec}$. The data were analysed by the $2^{-\Delta \Delta \mathrm{Cq}}$ method (21). For normalization, GAPDH was used as endogenous control to normalize lncRNA SNHG1 expression level and U6 was used as endogenous control to normalize miR-424-5p expression level. The sequences of the primers are as follows: SNHG1 forward, 5'-ACGTTGGAACCGAAGAGAGC-3' and reverse, 5'-GCA GCTGAATTCCCCAGGAT-3'; miR-424-5p forward, 5'-GGC TAGTCAGCAGCAATTCATGT-3' and reverse, 5'-GTGCAG GGTCCGAGGT-3'; FGF2 forward, 5'-AGGAGAGCGACC CACACATCAA-3' and reverse, 5'-AGCCAGCAGTCTTCC ATCTTCC-3'; U6 forward, 5'-CTCGCTTCGGCAGCACA-3' and reverse, 5'-AACGCTTCACGAATTTGCGT-3'; GAPDH forward, 5'-CCAGGTGGTCTCCTCTGACTT-3' and reverse, 5'-GTTGCTGTAGCCAAATTCGTTGT-3'.

MTT assay. Transfected MG63 and U2OS cells were seeded ( $2 \times 10^{5}$ cells/well) into a 96-well plate and incubated for 24 , 48, 72 and $96 \mathrm{~h}$. MTT (5 mg/ml; $20 \mu \mathrm{l}$; Sigma-Aldrich; Merck $\mathrm{KGaA}$ ) was added at different time points. After $2 \mathrm{~h}$ incubation at $37^{\circ} \mathrm{C}$, cell viability (optical density at $450 \mathrm{~nm}$ ) was analysed using a SpectraMax microplate spectrophotometer (Molecular Devices, LLC).

Wound healing assay. The transfected MG63 and U2OS cells $\left(2 \times 10^{5}\right.$ cells/well) were seeded into 6 -well plates. When the cells grew to a $100 \%$ confluence, wounds on the cell monolayer were created using a sterile p200 pipette tip, and the cells were incubated for $24 \mathrm{~h}$ in a serum-free medium. Subsequently, the cells were washed three times with PBS to wash away the floating cells. Images were captured at 0 and $24 \mathrm{~h}$ under a light microscope (magnification, $\mathrm{x} 400$; Olympus Corporation) and analysed with ImageJ software [version 1.46, National Institutes of Health (NIH)].

Transwell invasion assay. Cell invasion was assessed using Transwell chambers (Corning, Inc.) that were pre-coated (at $37^{\circ} \mathrm{C}$ for $30 \mathrm{~min}$ ) with Matrigel ${ }^{\circledR}$ (BD Biosciences). Transfected MG63 and U2OS cells ( $2 \times 10^{5}$ cells/well) were resuspended in serum-free medium and seeded into the Matrigel-coated upper chamber. A total of $600 \mu 1$ DMEM containing 10\% FBS was added into the lower chamber. After $24 \mathrm{~h}$ of culturing, the invasive cells were stained with $0.5 \%$ crystal violet. Invasive ability was evaluated by counting the number of invasive cells under a light microscope (magnification, $\mathrm{x} 400$; Olympus Corporation) in five randomly selected views.

Target prediction. StarBase version 2.0 (http://starbase. sysu.edu.cn), a software that decodes miRNA-ceRNA, miRNA-ncRNA and protein-RNA interaction networks from large-scale CLIP-Seq data, was used to predict the miRNA targets of SNHG1. A total of 144 putative targets was predicted. Among these miRNA targets, miR-424-5p was selected for the following assays owing to its important role in OS and the unknown regulatory relationship. In addition, TargetScan (http://www.targetscan.org), a software that predicts effective microRNA target sites in mammalian mRNAs, was used to predict the mRNA targets of miR-424-5p. Among the 1,515 target mRNAs, FGF2 was selected for the following assays owing to its important role in OS. 

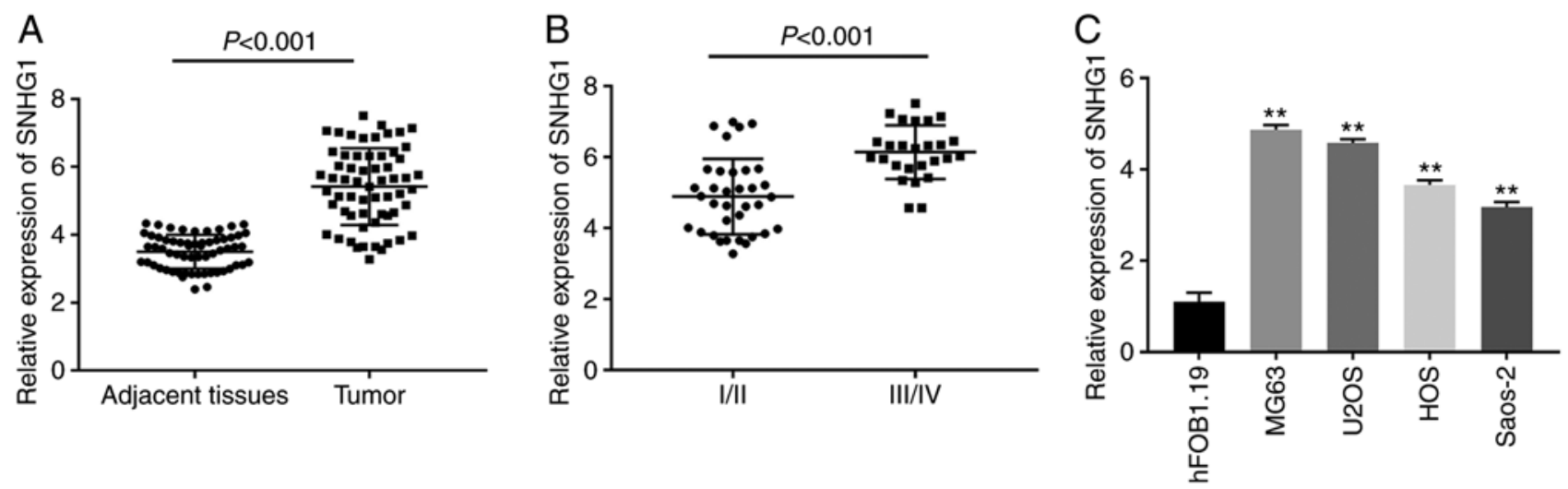

Figure 1. IncRNA SNHG1 expression is upregulated in OS tissues and cell lines. (A) RT-qPCR was used to detect the expression levels of lncRNA SNHG1 in OS and adjacent tissues. (B) The expression levels of SNHG1 were compared in patients with stage I/II and III/IV OS. (C) RT-qPCR was used to detect the expression levels of SNHG1 in hFOB1.19 and OS cell lines. The data were expressed as the mean $\pm \mathrm{SD}$. ${ }^{* *} \mathrm{P}<0.01 \mathrm{vs.} \mathrm{hFOB1.19}$. LncRNA, long non-coding RNA; OS, osteosarcoma; RT-qPCR, reverse transcription-quantitative PCR; SNHG1, small nucleolar RNA host gene 1.

RNA-binding protein immunoprecipitation assay (RIP). RIP was conducted using the EZ-Magna RIP RNA-Binding Protein Immunoprecipitation Kit (EMD Millipore). MG63 and U2OS cells $\left(5 \times 10^{5}\right.$ cells/well) were lysed with RIPA lysis buffer (Beyotime Institute of Biotechnology). Subsequently, the cell extracts were incubated with RIPA buffer magnetic beads as well as anti-Argonaute2 (AGO2) and anti-immunoglobulin G (IgG) (Shanghai Kanglang Biotechnology Co., Ltd.) at $4^{\circ} \mathrm{C}$ overnight and then washed with RIPA buffer (Beyotime Institute of Biotechnology). The eluates were collected, and the expression levels of SNHG1 and miR-424-5p were detected by RT-qPCR, aforementioned.

Dual-luciferase reporter gene (DLR) assay. The predicated binding sequences of SNHG1 (binding sites, $\underline{\mathrm{C}} \underline{\mathrm{A}}$ GUGAUGAAUUGCUGCU) and corresponding mutation sequences (므GGUGACUUAACGACGA) were inserted into the pGL3 vector to establish the SNHG1-wild-type (WT)/SNHG1-mutant-type (Mut). Similarly, The predicated binding sequences of FGF2 (binding sites, $\underline{A A A A U A}$ UUUUGCUGCU) and corresponding mutation sequences

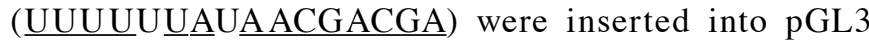
vector to construct the FGF2-WT/FGF2-Mut. MG63 and U2OS cells $\left(1 \times 10^{5}\right.$ cells/well) were then co-transfected with SNHG1-Mut/FGF2-Mut or SNHG1-WT/FGF2-WT (80 ng) and miR-424-5p mimics/miR-NC (50 nM) at $37^{\circ} \mathrm{C}$. After $48 \mathrm{~h}$ of culture, a Dual-Luciferase Reporter Assay System (Promega Corporation) was used to detect the luciferase activity. The activity of firefly luciferase was normalized to that of Renilla luciferase.

Western blot assay. The total protein from U2OS cells was extracted in RIPA buffer (Beyotime Institute of Biotechnology) containing $10 \mathrm{mmol} / \mathrm{l}$ phenylmethylsulfonyl fluoride (Beyotime Institute of Biotechnology); the protein concentration was detected by the BCA Protein Assay Kit (Abcam). All steps were conducted on ice. A total of $\sim 30 \mu \mathrm{g}$ protein was separated by $10 \%$ SDS-PAGE and then transferred to a polyvinylidene fluoride membrane (EMD Millipore). Membrane blocking was performed using 5\% bovine serum albumin (Thermo Fisher Scientific, Inc.) at room temperature.
Next, the membrane was incubated overnight at $4^{\circ} \mathrm{C}$ with primary antibodies against FGF2 (1:1,000; cat. no. ab208687; Abcam) and $\beta$-actin (1:1,000; cat. no. ab8226; Abcam). The membrane was washed with TBS + Tween-20 (0.05\%) three times followed by incubation with the HRP-conjugated rabbit anti-mouse IgG secondary antibody (1:3,000; cat. no. ab6728; Abcam) for $1 \mathrm{~h}$ at $37^{\circ} \mathrm{C}$. $\beta$-actin served as the internal loading control. Chemiluminescence was examined using the SuperSignal ${ }^{\text {TM }}$ West Femto Maximum Sensitivity Substrate (Thermo Fisher Scientific, Inc.) according to the manufacturer's protocol. ImageJ software (version 1.46; NIH) was utilised to semi-quantify the image.

Statistical analysis. SPSS Statistics 22.0 software (IBM Corp.) was used to analyse the data. The data are presented as the mean $\pm \mathrm{SD}$. The comparisons between two groups were analysed by unpaired t-tests, matched samples were compared by paired t-test, and the one-way ANOVA was measured for more than two groups. After ANOVA analysis, pairwise comparisons were assessed using Tukey's multiple comparisons test. Pearson's correlation analysis was used to determine the correlations between the expression of SNHG1 and miR-424-5p, FGF2 and miR-424-5p, as well as SNHG1 and FGF2 in OS tissues. $\mathrm{P}<0.05$ was considered to indicate a statistically significant difference. All experiments were conducted in triplicate in at least three independent experiments.

\section{Results}

lncRNA SNHG1 expression is significantly increased in OS tissues and cell lines. To evaluate whether SNHG1 influences the development of OS, samples from 61 patients with OS were obtained and compared with the adjacent normal tissues. The expression levels of SNHG1 in OS tissues was found to be significantly higher compared with the adjacent tissues $(\mathrm{P}<0.001$; Fig. 1A). In addition, it was also determined that SNHG1 expression in OS is related to its clinical stages. The expression of SNHG1 in stage III/IV of OS was significantly higher compared with the expression levels in stage I/II, which indicated that SNHG1 expression was related to the severity of OS $(\mathrm{P}<0.001$; Fig. 1B). As presented in Table I, the patients 
Table I. Clinicopathological characteristics of patients with OS, and lncRNA SNHG1 expression levels in OS tissues.

\begin{tabular}{|c|c|c|c|c|}
\hline \multirow[b]{2}{*}{ Clinicopathological characteristic } & \multirow[b]{2}{*}{$\mathrm{n}=61$} & \multicolumn{2}{|c|}{ lncRNA SNHG1 expression } & \multirow[b]{2}{*}{ P-value } \\
\hline & & Low $(n=30)$ & $\operatorname{High}(\mathrm{n}=31)$ & \\
\hline Age, years & & & & 0.699 \\
\hline$<20$ & 31 & 16 & 15 & \\
\hline$\geq 20$ & 30 & 14 & 16 & \\
\hline Sex & & & & 0.885 \\
\hline Male & 27 & 13 & 14 & \\
\hline Females & 34 & 17 & 17 & \\
\hline Diameter, $\mathrm{cm}$ & & & & 0.683 \\
\hline$<3$ & 26 & 12 & 14 & \\
\hline$\geq 3$ & 35 & 18 & 17 & \\
\hline Resection degree & & & & 0.699 \\
\hline Total resection & 31 & 16 & 15 & \\
\hline Subtotal resection & 30 & 14 & 16 & \\
\hline WHO Grade & & & & $<0.001$ \\
\hline $\mathrm{I}+\mathrm{II}$ & 35 & 24 & 11 & \\
\hline III + IV & 26 & 6 & 20 & \\
\hline
\end{tabular}

lncRNA SNHG1, long non-coding RNA small nucleolar RNA host gene 1; OS, osteosarcoma.

A
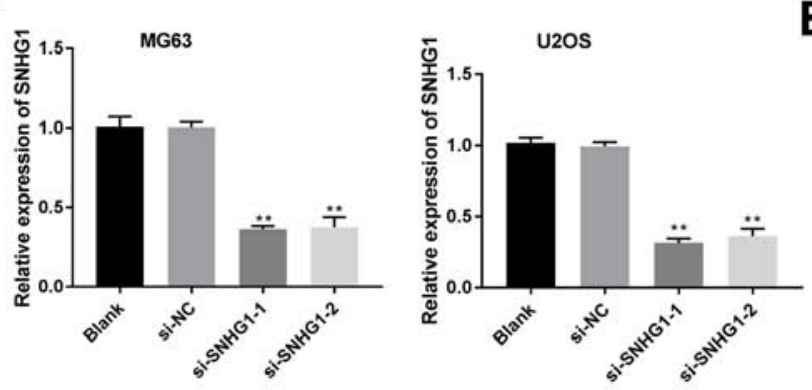

C
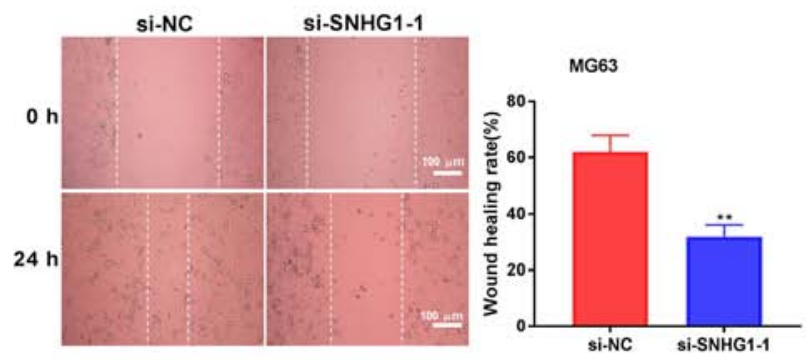

D
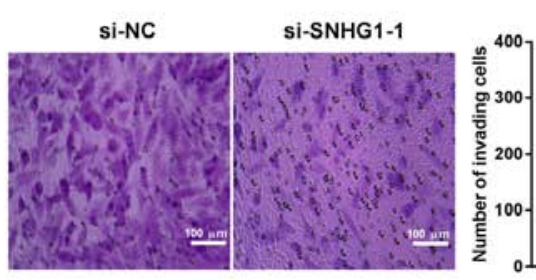

MG63

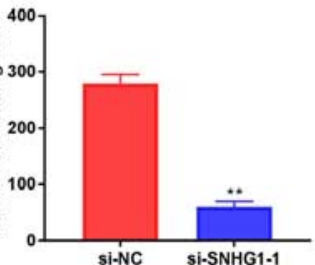

B
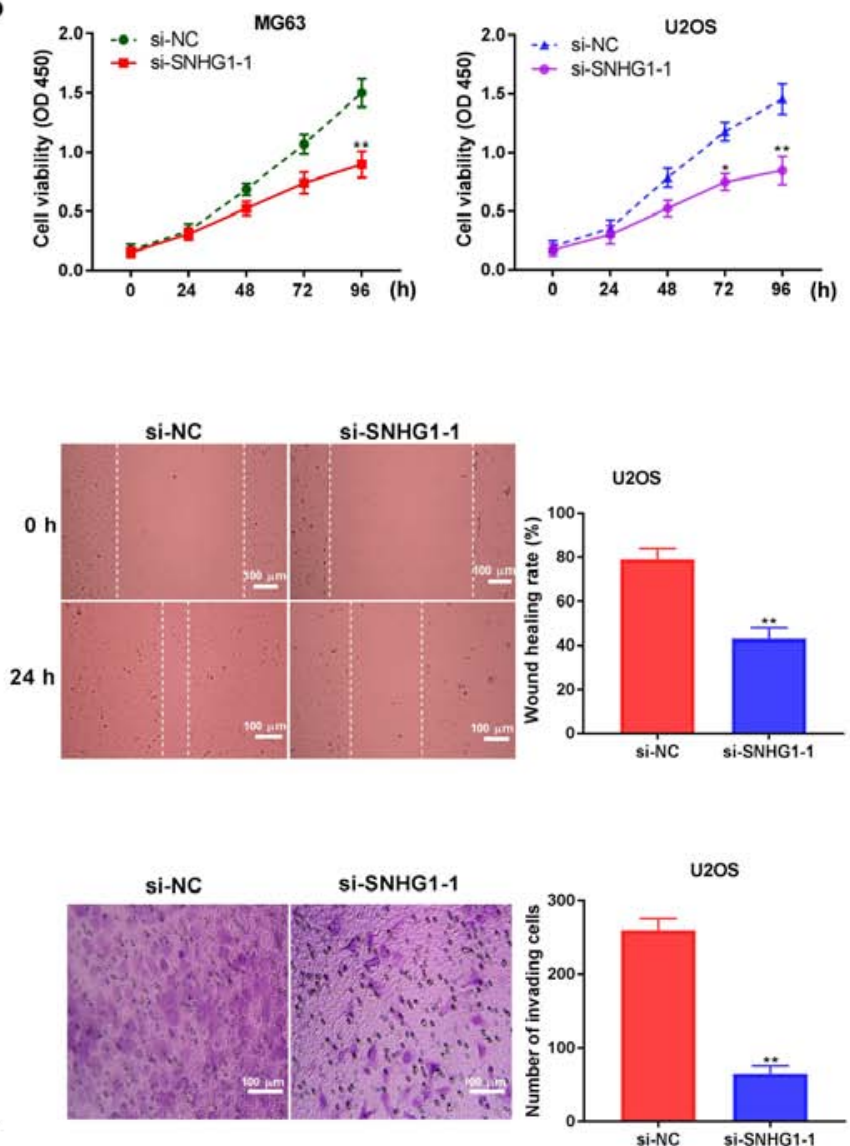

Figure 2. IncRNA SNHG1 knockdown inhibits proliferation, migration and invasion of OS cells. (A) Reverse transcription-quantitative PCR was used to detect the expression of lncRNA SNHG1 after transfection of si-SNHG1-1, si-SNHG1-2 and si-NC into MG63 and U2OS OS cells. (B) The viability of OS cells was detected by MTT assay. (C) The migratory ability of OS cells was determined by wound healing assay. (D) The invasive ability of OS cells was analyzed by Transwell invasion assay. Scale bar, $100 \mu \mathrm{m}$; magnification, $\mathrm{x} 400$. The data are expressed as the mean $\pm \mathrm{SD}$. ${ }^{*} \mathrm{P}<0.05$, $^{* *} \mathrm{P}<0.01 \mathrm{vs}$. si-NC. LncRNA, long non-coding RNA; NC, negative control; OD, optical density; OS, osteosarcoma; si-; small interfering RNA; SNHG1, small nucleolar RNA host gene 1. 
A

SNHG1 WT

miR-424-5p

SNHG1 Mut

\section{5' caCCAGUGAUGAAUUGCUGCUc 3 \\ | | |||||||||||| \\ 3' aaGUUUUGUACUUAACGACGAC 5'}

5' caGCUGUGUACUUAACGACGAC 3'
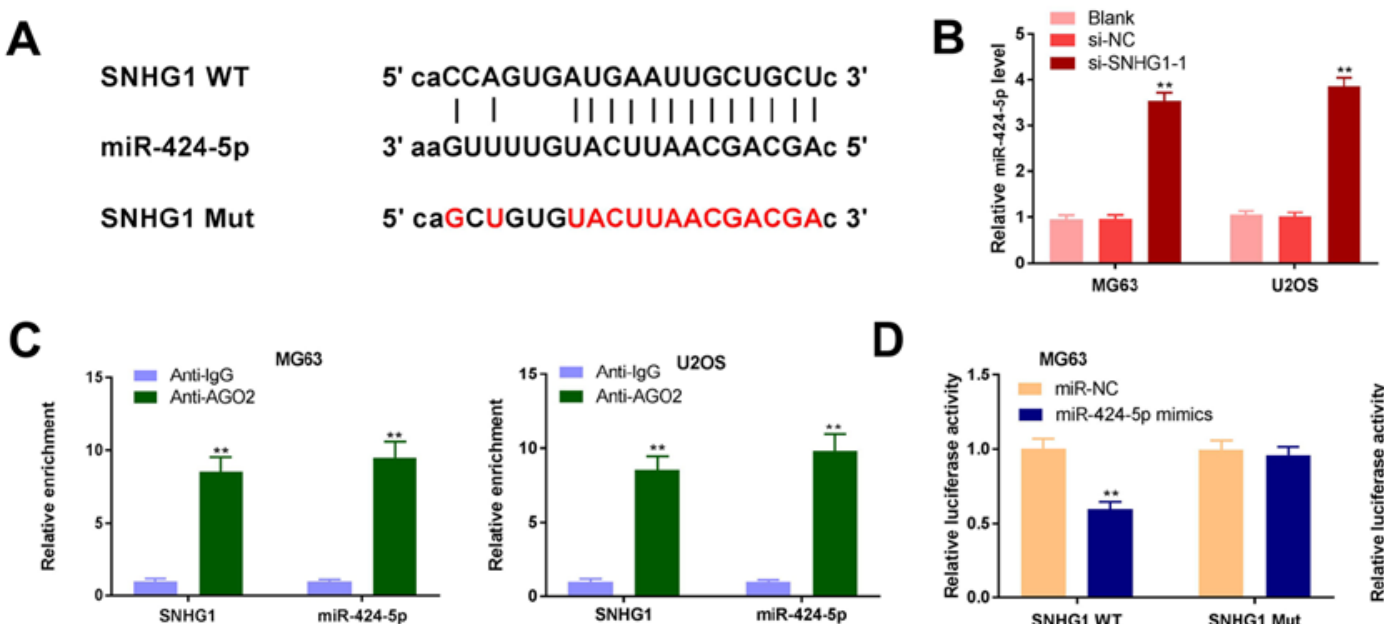

D
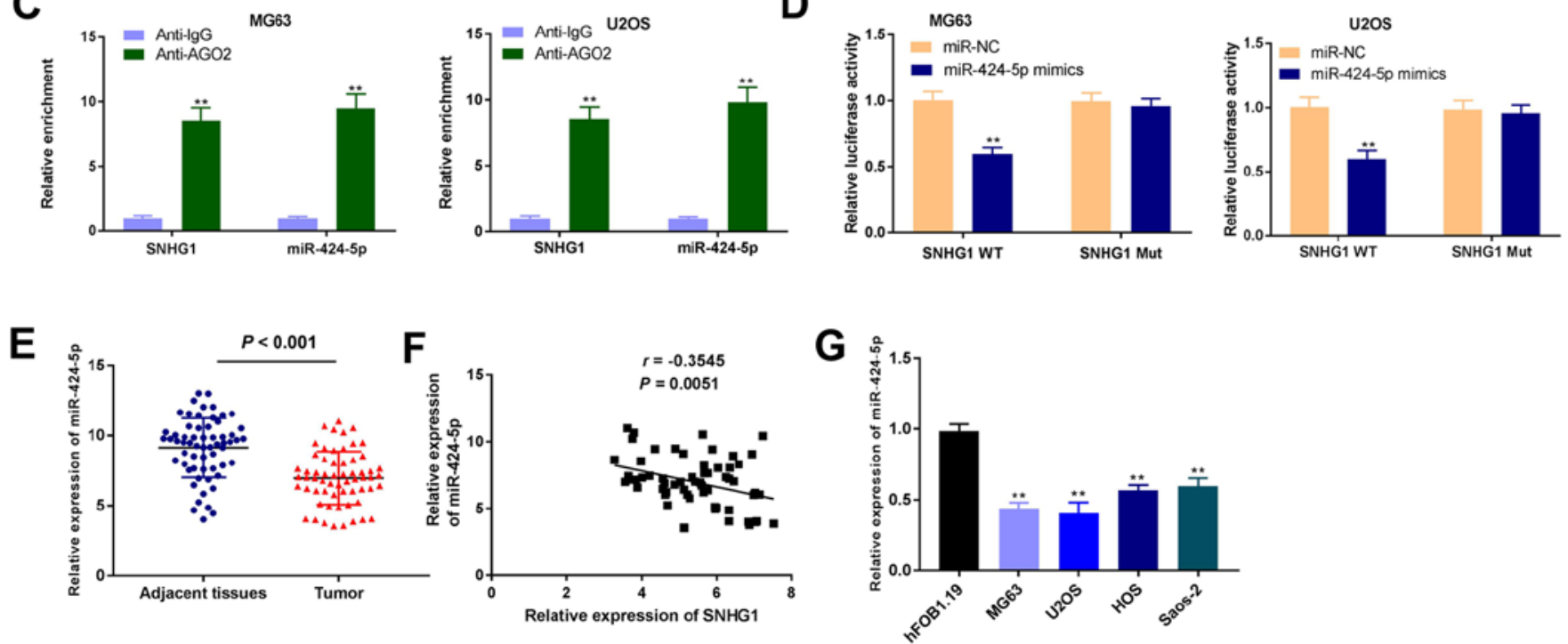

Figure 3. IncRNA SNHG1 targets miR-424-5p. (A) starBase was used to predict the binding between lncRNA SNHG1 and miR-424-5p. (B) RT-qPCR was used to detect the expression levels of miR-424-5p after transfection of si-SNHG1-1 into MG63 and U2OS OS cells. ${ }^{* *} \mathrm{P}<0.01$ vs. si-NC. (C) RNA-binding protein immunoprecipitation assay was performed in OS cells, and the expression of SNHG1 and miR-424-5p was detected by RT-qPCR. ${ }^{* * *} \mathrm{P}<0.01$ vs. Anti-IgG. (D) Dual-luciferase reporter gene assays were used to confirm the targeting relationship between SNHG1 and miR-424-5p. ${ }^{* *} \mathrm{P}<0.01 \mathrm{vs.} \mathrm{miR-NC.} \mathrm{(E)} \mathrm{RT-qPCR}$ was used to detect the expression of miR-424-5p in patient OS and adjacent normal tissues. (F) Correlation analysis between SNHG1 and miR-424-5p. (G) RT-qPCR was used to detect the expression of miR-424-5p in hFOB1.19 and OS cell lines. ${ }^{* *} \mathrm{P}<0.01 \mathrm{vs.hFOB1.19}$. The data are expressed as the mean \pm SD. AGO2, argonaute2; IgG, immunoglobulin G; lncRNA, long non-coding RNA; miR, microRNA; Mut, mutant; NC, negative control; OS, osteosarcoma; RT-qPCR, reverse transcription-quantitative PCR; si, small interfering RNA; SNHG1, small nucleolar RNA host gene 1; WT, wild-type.

were divided into two groups: High and low lncRNA SNHG1 expression, using the median expression level as the cut-off point. High and low expression levels of SNHG1 were speculated to exhibit distinct differences based on tumour stage. SNHG1 expression levels were also detected in hFOB1.19 and a number of OS cell lines. SNHG1 was found to be expressed in the four OS cell lines at significantly higher levels compare with expression in the hFOB1.19 cells ( $\mathrm{P}<0.01$; Fig. 1C). MG63 and $\mathrm{U} 2 \mathrm{OS}$ cell lines were selected for the further experiments due to their relatively high expression of SNHG1. These results indicated that SNHG1 may be an onco-lncRNA in OS.

Silencing lncRNA SNHG1 inhibits the proliferation, migration and invasion of OS cells. Following transfection of si-SNHG1-1, si-SNHG1-2 and si-NC into OS cells, SNHG1 expression level was detected by RT-qPCR. The results indicated that SNHG1 expression in MG63 and U2OS cells were downregulated after transfection with si-SNHG1-1 and SNHG1-2 compared with the si-NC group ( $\mathrm{P}<0.01$; Fig. $2 \mathrm{~A})$. Using the MTT assay, it was confirmed that the viability was significantly inhibited 96 and $72 \mathrm{~h}$ after si-SNHG1-1 transfection in MG63 and U2OS cells, respectively $(\mathrm{P}<0.05$; Fig. $2 \mathrm{~B})$. The migratory ability of the $\mathrm{OS}$ cell lines was also significantly inhibited after SNHG1 knockdown compared with si-NC ( $\mathrm{P}<0.01$; Fig. 2C). The Transwell invasion assay showed similar results; the invasive ability of
OS cells was significantly inhibited after SNHG1 interference $(\mathrm{P}<0.01$; Fig. 2D). Together, these data demonstrated that SNHG1 silencing may limit the proliferation, migration and invasion of OS cells.

lncRNA SNHG1 targets miR-424-5p. Using starBase software, the binding region between miR-424-5p and SNHG1 was predicted (Fig. 3A). miR-424-5p expression levels were detected after transfection of si-SNHG1-1 into MG63 and U2OS OS cells, and the results revealed that the expression of miR-424-5p was significantly increased in the si-SNHG1-1 group compared with the si-NC group $(\mathrm{P}<0.01$; Fig. $3 \mathrm{~B})$. The RIP assay demonstrated that in OS cell lines, SNHG1 was enriched with anti-AGO2 compared with those of the anti-IgG control and that miR-424-5p exhibited similar results $(\mathrm{P}<0.01$; Fig. 3C). The DLR assays showed a marked decrease in luciferase activity in the SNHG1 WT + miR-424-5p mimics group compared with that of the SNHG1 WT + miR-NC group $(\mathrm{P}<0.01$; Fig. 3D). miR-424-5p expression in the patient tumour and adjacent tissues were also detected; RT-qPCR results demonstrated that miR-424-5p expression levels in OS tissues decreased significantly compared with expression levels in the adjacent tissues $(\mathrm{P}<0.001$; Fig. $3 \mathrm{E})$. Correlation analysis between SNHG1 and miR-424-5p expression levels revealed that there was a negative correlation $(r=-0.3545 ; \mathrm{P}=0.0051$; 
A
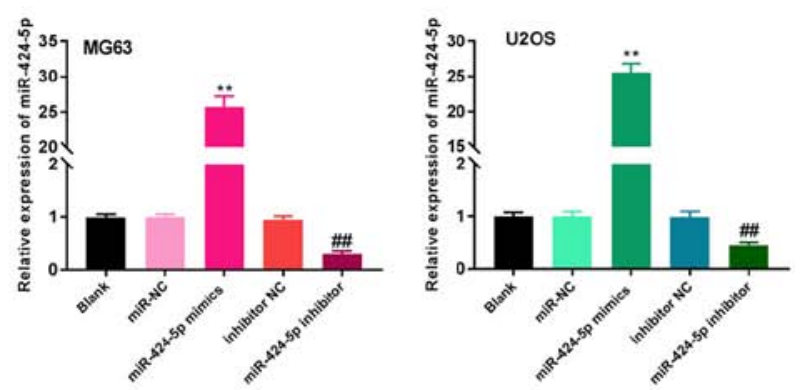

B
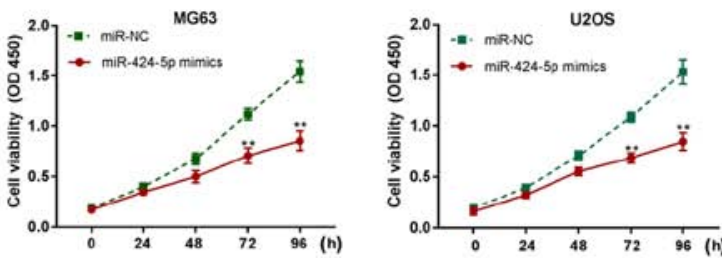

C
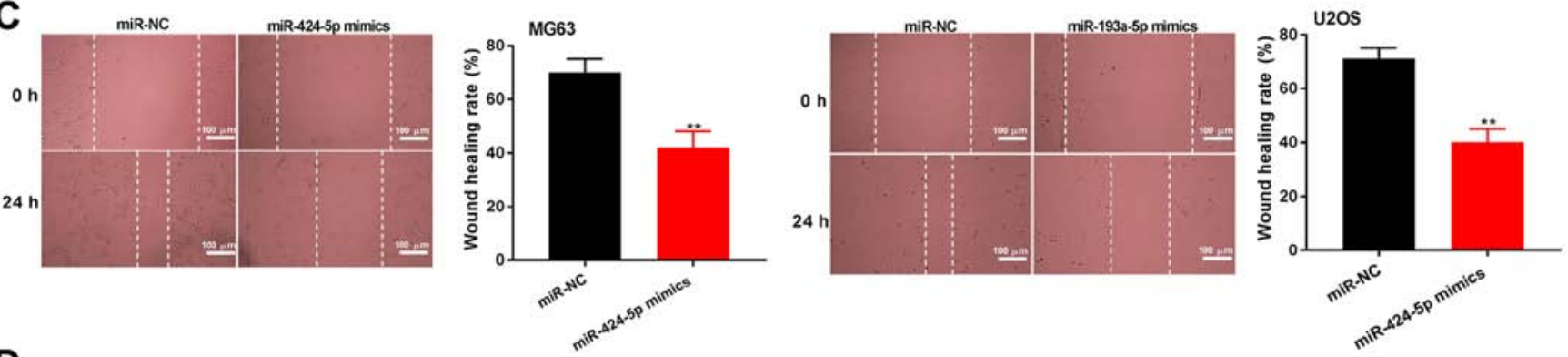

D
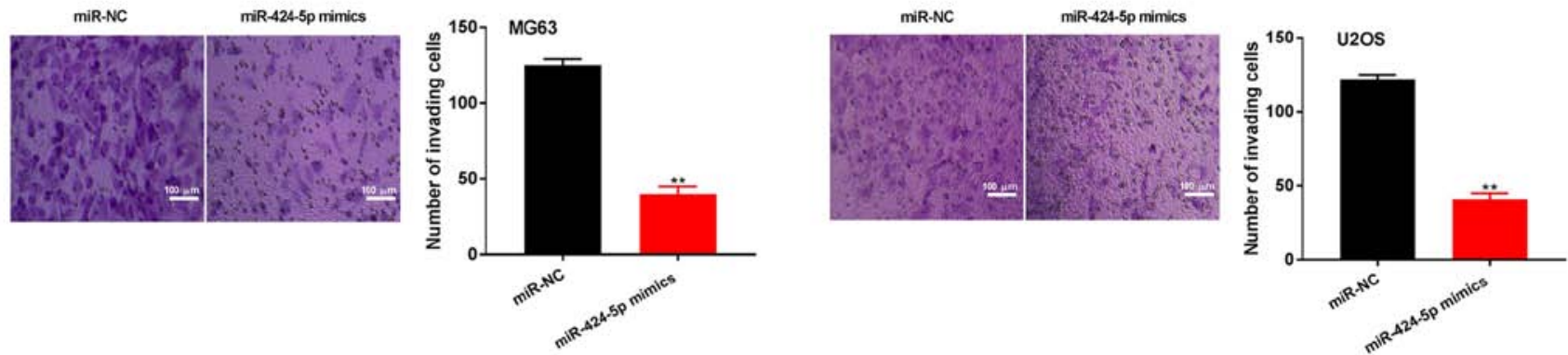

Figure 4. Overexpression of miR-424-5p inhibits the proliferation, migration and invasion of OS cells. (A) Reverse transcription-quantitative PCR was used to detect the expression levels of miR-424-5p after transfection of miR-424-5p mimics, miR-NC, miR-424-5p inhibitor or inhibitor NC into MG63 and U2OS OS cells. (B) The viability of OS cells was detected by MTT assay. (C) Wound healing assays were used to determine the migratory ability of OS cells. (D) Transwell invasion assays were used to analyze the invasion ability of OS cells. Scar bar, $100 \mu \mathrm{m}$; magnification x400. The data are expressed as the mean \pm SD. ${ }^{* *} \mathrm{P}<0.01$ vs. miR-NC; ${ }^{\# \#} \mathrm{P}<0.01$ vs. inhibitor NC. miR, microRNA; NC, negative control; OD, optical density; OS, osteosarcoma.

Fig. 3F). Finally, the expression of miR-424-5p was detected in OS and normal human osteoblast cell lines. RT-qPCR results indicated that miR-424-5p expression levels in the OS cells were significantly reduced compared with that of hFOB1.19 $(\mathrm{P}<0.01$; Fig. 3G). These data indicated that miR-424-5p was the target of, and negatively modulated by, SNHG1.

miR-424-5p upregulation limits the proliferation, migration and invasion of OS cells. After transfection of miR-424-5p mimics, miR-NC, miR-424-5p inhibitor or inhibitor NC into MG63 and U2OS OS cells, miR-424-5p expression was detected. The results demonstrated that the expression of miR-424-5p was upregulated after transfection of miR-424-5p mimics and downregulated after transfection of the miR-424-5p inhibitor, compared with the respective controls $(\mathrm{P}<0.01$; Fig. 4A). The MTT assay revealed that miR-424-5p overexpression significantly suppressed the viability of OS cells at $72 \mathrm{~h}(\mathrm{P}<0.01$; Fig. 4B). Furthermore, the wound healing assay confirmed that upregulated miR-424-5p expression significantly inhibited the migratory ability of OS cells $(\mathrm{P}<0.01$; Fig. 4C). miR-424-5p overexpression also limited the number of invasive OS cells $(\mathrm{P}<0.01$; Fig. 4D). These results indicated that overexpression of miR-424-5p inhibits the proliferation, migration and invasion of OS cells.
miR-424-5p targets FGF2. Using TargetScan software, FGF2 was predicted to be a downstream target of miR-424-5p (Fig. 5A). The DLR assay revealed that in MG63 and U2OS OS cell lines, the luciferase activity in the FGF2 WT + miR-424-5p mimics group was significantly decreased compared with that of the FGF2 WT + miR-NC group, an effect that was partially reversed when SNHG1 was overexpressed $(\mathrm{P}<0.01$; Fig. 5B). In addition, the expression levels of FGF2 in patient tissue samples were examined; FGF2 expression levels in the OS tissues were significantly higher compared with expression in the adjacent normal tissues $(\mathrm{P}<0.001$; Fig. 5C). Correlation analysis indicated that FGF2 expression was negatively correlated with miR-424-5p expression $(\mathrm{r}=-0.5258 ; \mathrm{P}<0.0001$; Fig. 5D) and positively correlated with SNHG1 expression $(\mathrm{r}=0.5196 ; \mathrm{P}<0.0001$; Fig. 5E). Finally, compared with the hFOB1.19 cells group, the expression of FGF2 in the OS cell lines were found to be significantly higher $(\mathrm{P}<0.01$; Fig. $5 \mathrm{~F})$. The aforementioned data suggested that FGF2, which was highly expressed in OS, is a target of miR-424-5p.

SNHG1 knockdown inhibits the proliferation, migration and invasion of $O S$ cells by regulating $m i R-424-5 p / F G F 2$ in vitro. The transfection efficiency of pcDNA-SNHG1 or pcDNA-FGF2 in U2OS cells was initially determined; the 
B

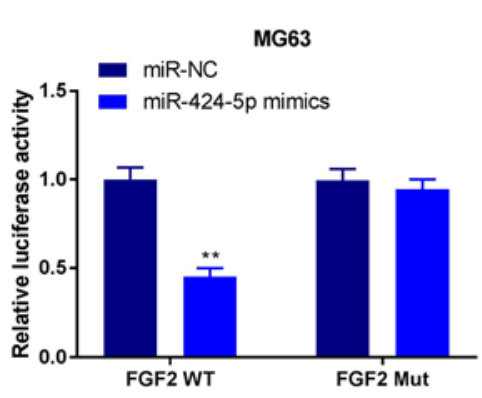

D

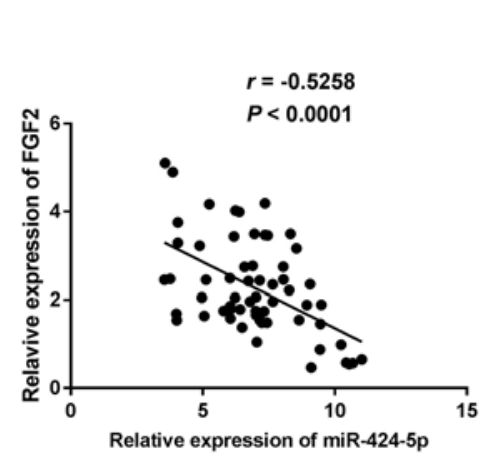

U2OS

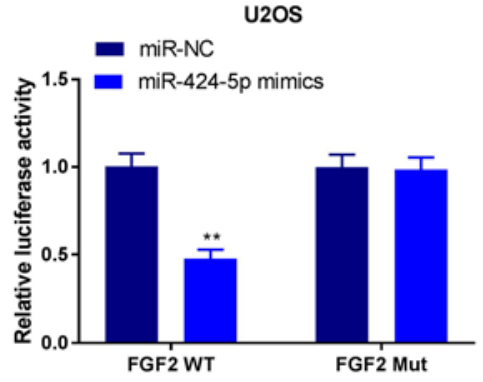

E

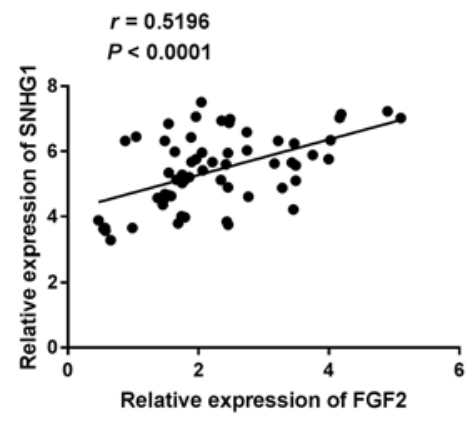

C

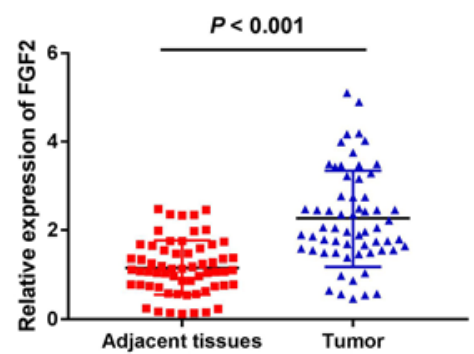

$F$

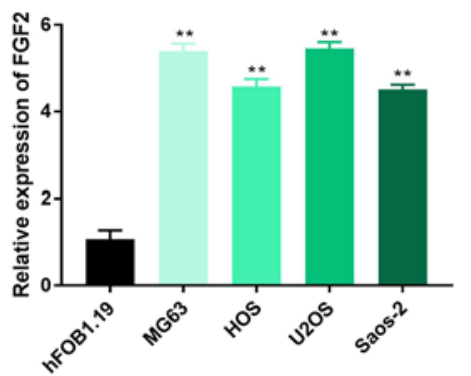

Figure 5. miR-424-5p targets FGF2. (A) TargetScan was used to predict target sites between the miR-424-5p and FGF2. (B) DLR assays were used to confirm the targeting relationship. ${ }^{* *} \mathrm{P}<0.01$ vs. miR-NC + pcDNA-NC group. (C) RT-qPCR was used to determine the expression levels of FGF2 in patient OS and adjacent tissues. (D) Correlation analysis between FGF2 and miR-424-5p. (E) Correlation analysis between SNHG1 and FGF2. (F) RT-qPCR was used to detect the expression levels of FGF2 in hFOB1.19 and OS cell lines. ${ }^{* *} \mathrm{P}<0.01$ vs. hFOB1.19. The data are expressed as the mean \pm SD. DLR, dual-luciferase reporter gene; FGF2, fibroblast growth factor-2; miR, microRNA; Mut, mutant; NC, negative control; RT-qPCR, reverse transcription-quantitative PCR; SNHG1, small nucleolar RNA host gene 1; WT, wild-type.

expression levels of SNHG1 were significantly increased by transfection of pcDNA-SNHG1 in U2OS cells $(\mathrm{P}<0.01$; Fig. 6A), and FGF2 expression levels were significantly upregulated in U2OS cells transfected with pcDNA-FGF2 $(\mathrm{P}<0.01$; Fig. 6B). Western blot analysis results demonstrated that miR-424-5p overexpression in U2OS cells significantly inhibited FGF2 expression, which could be partially reversed by overexpression of SNHG1 ( $\mathrm{P}<0.01$; Fig. 6C). The MTT assay revealed that cell viability was inhibited by depletion of SNHG1 at $96 \mathrm{~h}$, but this effect could be partly reversed by miR-424-5p inhibition or overexpression of FGF2 $(\mathrm{P}<0.01$; Fig. 6D). Similarly, the wound healing and Transwell invasion assays demonstrated that inhibition of SNHG1 suppressed the migratory and invasive abilities of U2OS cells, both of which could be reversed by miR-424-5p downregulation and FGF2 upregulation $(\mathrm{P}<0.01$; Fig. $6 \mathrm{E}$ and $\mathrm{F})$. These data indicated that SNHG1 knockdown may suppress the proliferation, migration and invasion of OS cells by regulating the miR-424-5p/FGF2 axis (Fig. 7).

\section{Discussion}

$\mathrm{OS}$ is one of the most common malignant bone tumours in adolescents (22). Several lncRNAs have been reported to be involved in the regulation of OS. For example, Fei et al (16) discovered that expression of the lncRNA regulator of reprogramming (ROR) is significantly increased in OS tissues and in OS cell lines, and that upregulation of ROR appears strongly related to tumour stage. Yang et al (23) found that the expression of IncRNA XIST is dramatically elevated in OS tissues and also strongly associated with tumour stage. Similarly, results from the present study demonstrated that SNHG1 expression is elevated in OS tissues and cell lines, and presents a notable correlation with tumour stage. Thus, it was hypothesised that SNHG1 may act as a pathogenic factor in OS.

In the past decade, researchers have determined that lncRNAs function as crucial regulators in the progression of OS. For example, Li et al (9) reported that suppression of XIST limits the proliferation and invasion of OS cells. Zhao et al (24) discovered that ASBEL interference decreases viability and the migratory and invasive abilities of OS cells. Xu et al (25) found that SNHG4 inhibition suppresses the proliferation of OS cells. The present study found that knockdown of SNHG1 inhibited the viability, migratory and invasive abilities of OS cells. Similar findings in another study demonstrated that OS cell viability, migration and invasion is regulated by silencing SNHG1, thereby eventually halting the progression of OS (14). 
A

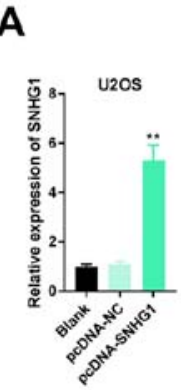

B

E

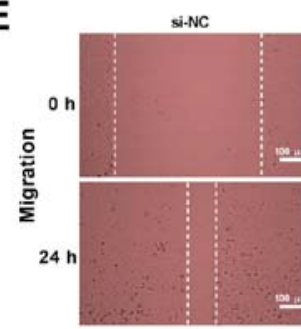

C

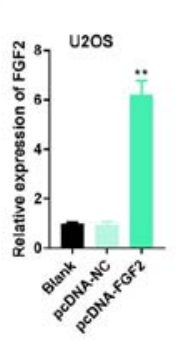

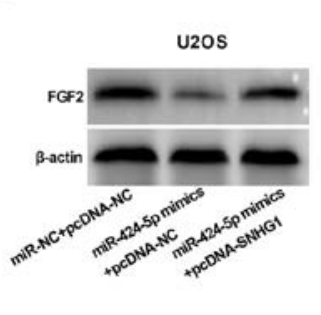

- miR-NC+DCDNA-NC = miR-424-5p mimics+pCDNA-NC $1.5=$ miR-424-5P mimics+PCDNA-SNHG1

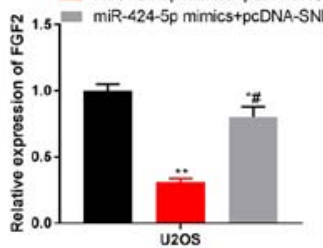

D

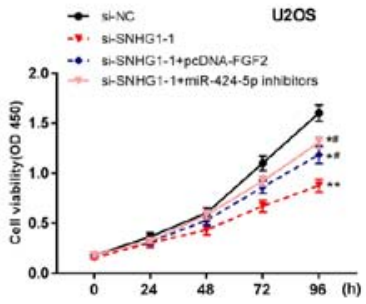

$\mathbf{F}$
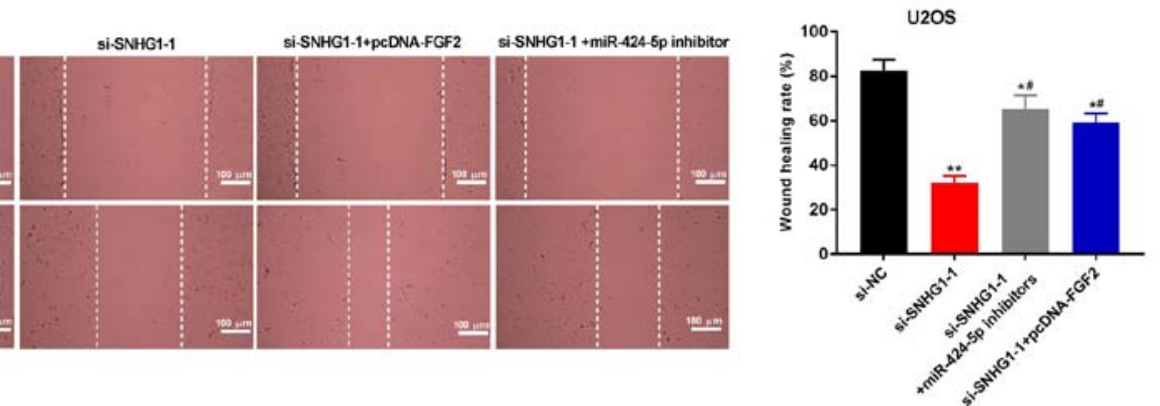

U2OS

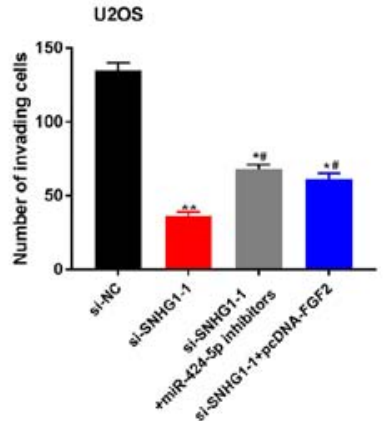

Figure 6. IncRNA SNHG1 knockdown inhibits proliferation, migration and invasion of U2OS cells by regulating miR-424-5p/FGF2. (A) RT-qPCR was used to detect the expression levels of SNHG1 after transfection of pcDNA-SNHG1. ${ }^{* *} \mathrm{P}<0.01 \mathrm{vs}$. pcDNA-NC. (B) RT-qPCR was used to detect the expression of

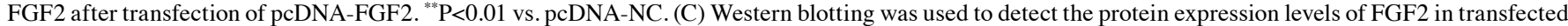
U2OS cells. "P<0.05, ${ }^{* *} \mathrm{P}<0.01$ vs. miR-NC + pcDNA-NC; ${ }^{~} \mathrm{P}<0.05$ vs. miR-424-5p mimics + pcDNA-NC. (D) MTT assays were used to detect the viability of transfected U2OS cells. ${ }^{*} \mathrm{P}<0.05,{ }^{* *} \mathrm{P}<0.01$ vs. si-NC; ${ }^{~} \mathrm{P}<0.05$ vs. si-SNHG1-1. (E) Wound healing assays were used to determine the migratory ability of transfected U2OS cells. ${ }^{*} \mathrm{P}<0.05,{ }^{* * *} \mathrm{P}<0.01$ vs. si-NC; ${ }^{~} \mathrm{P}<0.05$ vs. si-SNHG1-1. (F) Transwell invasion assays were used to analyze the invasive ability of transfected U2OS cells. Scar bar, $100 \mu \mathrm{m}$; magnification, $\mathrm{x} 400 .{ }^{*} \mathrm{P}<0.05,{ }^{* *} \mathrm{P}<0.01$ vs. si-NC; ${ }^{*} \mathrm{P}<0.05$ vs. si-SNHG1-1. The data are expressed as the mean \pm SD. FGF2, FGF2, fibroblast growth factor-2; lncRNA, long non-coding RNA; miR, microRNA; NC, negative control; OD, optical density; OS, osteosarcoma; si-, small interfering RNA; SNHG1, small nucleolar RNA host gene 1.

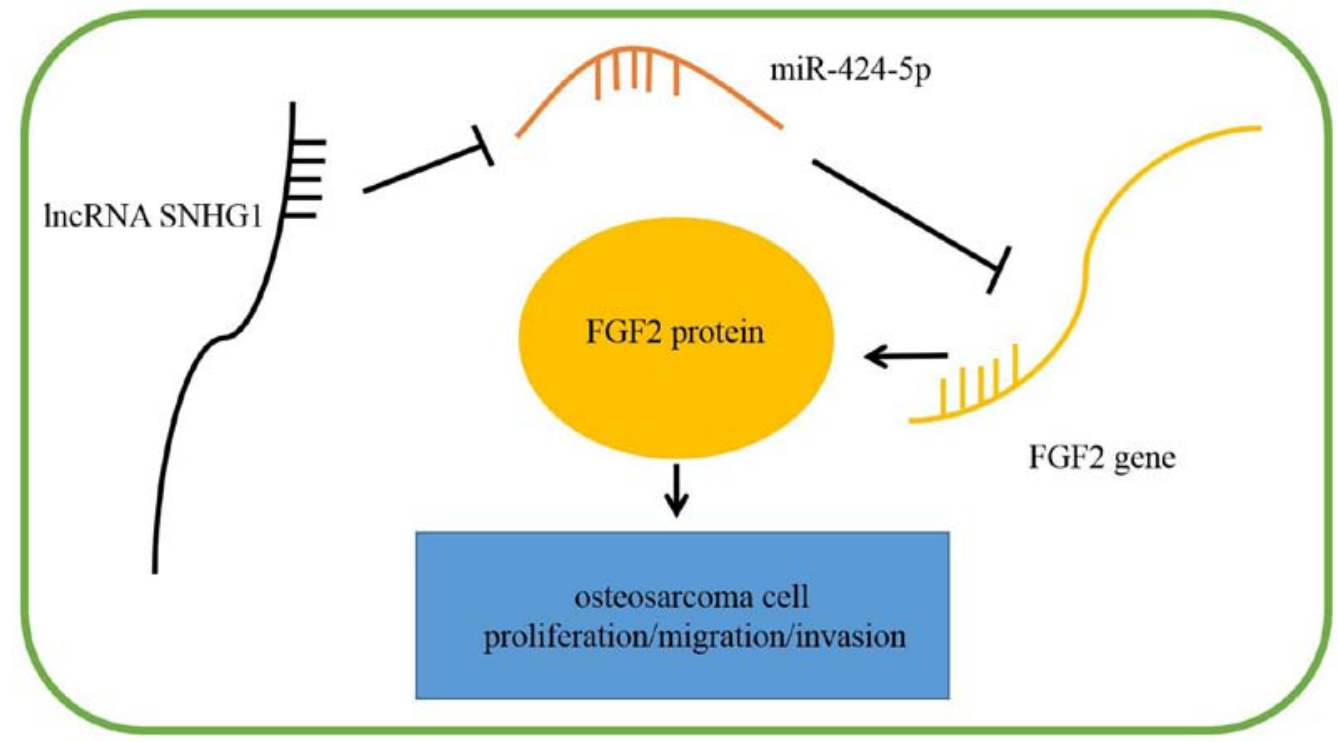

Figure 7. Schematic diagram of the regulatory mechanism of the SNHG1/miR-424-5p/FGF2 axis in osteosarcoma cells. FGF2, fibroblast growth factor-2; lncRNA, long non-coding RNA; miR, microRNA; SNGH1, small nucleolar RNA host gene 1. 
Therefore, it was hypothesized that SNHG1 inhibition may attenuate the development of OS.

miR-424 has been shown to attenuate the progression of several cancers, including endometrial cancer (EC) (26), breast cancer (BC) (27) and colorectal cancer(CRC) (28). Dong et al (26) reported that miR-424 expression is decreased in EC tissues and cell lines, and that upregulation of miR-424 suppresses EC cell invasion. Wang et al (27) found that miR-424 is minimally expressed in BC tissues and cell lines, and miR-424 upregulation inhibits the development of BC. Fang et al (28) demonstrated that miR-424 expression in CRC tissues and cell lines is low, and overexpression of miR-424 eventually suppresses the growth of CRC. The present study discovered that miR-424-5p expression is significantly lower in OS tissues and cell lines. Furthermore, OS cell viability, migration and invasion are inhibited by miR-424-5p overexpression. Consistent with these results, previous studies have reported that miR-424 expression is decreased in OS tissues and that miR-424 overexpression limits the viability (29), as well as the migratory and invasive abilities (17) of OS cells. In addition, the present study further determined that miR-424-5p is the target of and negatively modulated by SNHG1. Therefore, it was hypothesized that miR-424-5p may be regulated by SNHG1 to inhibit the development of OS.

FGF2 is a member of the FGF family and has been thought to take part in the development of various cancers (30-32). Zhang et al (33) found that FGF2 is highly expressed in cervical carcinoma tissues, and Cheng et al (34) reported that FGF2 expression is elevated in non-small cell lung cancer tissues and cells. The present study showed that FGF2 expression is upregulated in OS tissues and cell lines. Similarly, Sun et al (35) observed that FGF2 expression in OS tissues is significantly upregulated. In addition, the present study demonstrated that miR-424-5p targeted FGF2, and there is a notable inverse correlation between their expressions. The results indicated that FGF2 may be negatively modulated by miR-424-5p. The present study also found that high expression of miR-424-5p and low expression of FGF2 both reverse the suppressive effects of SNHG1 knockdown on the viability, migratory and invasive abilities of OS cells. These data indicated that SNHG1 knockdown may inhibit the viability, migration and invasion of OS cells by regulating miR-424-5p/FGF2.

However, there are some limitations within the present study. For instance, only miR-424-5p mimics were used in cell culture; miR-424-5p inhibitor should be added in future studies to further support these results. Secondly, the regulatory effects of the SNHG1/miR-424-5p/FGF2 axis on the apoptosis of OS cells was not determined. Furthermore, the present study was limited to in vitro experiments, thus, additional research using animal models is required. Finally, the detailed mechanisms of the SNHG1/miR-424-5p/FGF2 axis on OS, such as upstream factors and related signalling pathways, need to be further explored.

Collectively, results from the presented study suggested that SNHG1 knockdown may suppress the progression of OS by regulating miR-424-5p/FGF2 in vitro. Thus, the SNHG1/miR-424-5p/FGF2 axis may present a new potential target for treating OS.

\section{Acknowledgements}

Not applicable.

\section{Funding}

The present work is supported by the Featured Clinical Discipline Project of Shanghai Pudong (grant no. PWYts2018-2) and the construction of key discipline group of Sanitary System of Shanghai Pudong New District (grant no. PWZxq2017 12).

\section{Availability of data and materials}

The datasets used and/or analyzed during the current study are available from the corresponding author on reasonable request.

\section{Authors' contributions}

ZL made substantial contributions to the conception and design of the study. ZL, XW, and SL made substantial contributions to the acquisition, analysis and interpretation of data, as well as the drafting and revision of the manuscript. All authors confirmed the authenticity of all the raw data, gave final approval of the version to be published, and agreed to be accountable for all aspects of the work.

\section{Ethics approval and consent to participate}

The present study was conducted after obtaining local Ethical Committee approval of Zibo Zhoucun People's Hospital. Written informed consent was obtained from patients.

\section{Patient consent for publication}

Not applicable.

\section{Competing interests}

The authors declare that they have no competing interests.

\section{References}

1. Mirabello L, Troisi RJ and Savage SA: International osteosarcoma incidence patterns in children and adolescents, middle ages and elderly persons. Int J Cancer 125: 229-234, 2009.

2. Kumar R, Kumar M, Malhotra K and Patel S: Primary osteosarcoma in the elderly revisited: Current concepts in diagnosis and treatment. Curr Oncol Rep 20: 13, 2018.

3. Harrison DJ, Geller DS, Gill JD, Lewis VO and Gorlick R: Current and future therapeutic approaches for osteosarcoma. Expert Rev Anticancer Ther 18: 39-50, 2018.

4. Shaikh AB, Li F, Li M, He B, He X, Chen G, Guo B, Li D, Jiang F, Dang L, et al: Present advances and future perspectives of molecular targeted therapy for osteosarcoma. Int J Mol Sci 17: $506,2016$.

5. Zhang B, Zhang Y, Li R, Li J, Lu X and Zhang Y: The efficacy and safety comparison of first-line chemotherapeutic agents (high-dose methotrexate, doxorubicin, cisplatin, and ifosfamide) for osteosarcoma: A network meta-analysis. J Orthop Surg Res 15: 51,2020.

6. Luetke A, Meyers PA, Lewis I and Juergens H: Osteosarcoma treatment-where do we stand? A state of the art review. Cancer Treat Rev 40: 523-532, 2014.

7. Rinn JL and Chang HY: Genome regulation by long noncoding RNAs. Annu Rev Biochem 81: 145-166, 2012. 
8. Zhang Q, Geng PL, Yin P, Wang XL, Jia JP and Yao J: Down-regulation of long non-coding RNA TUG1 inhibits osteosarcoma cell proliferation and promotes apoptosis. Asian Pac J Cancer Prev 14: 2311-2315, 2013.

9. Li H, Cui J, Xu B, He S, Yang H and Liu L: Long non-coding RNA XIST serves an oncogenic role in osteosarcoma by sponging miR-137. Exp Ther Med 17: 730-738, 2019.

10. Liu P, He W, Lu Y and Wang Y: Long non-coding RNA LINC00152 promotes tumorigenesis via sponging miR-193b-3p in osteosarcoma. Oncol Lett 18: 3630-3636, 2019.

11. Zhang H, Lu Y, Wang J, Zhang T, Dong C, Li X, Wang X, Ma Q, Yang T and Zhou Y: Downregulation of the long non-coding RNA FOXD2-AS1 inhibits cell proliferation, migration and invasion in osteosarcoma. Mol Med Rep 20: 292-302, 2019.

12. Deng R, Zhang J and Chen J: lncRNA SNHG1 negatively regulates miRNA-101-3p to enhance the expression of ROCK1 and promote cell proliferation, migration and invasion in osteosarcoma. Int J Mol Med 43: 1157-1166, 2019.

13. Jiang Z, Jiang $C$ and Fang J: Up-regulated lnc-SNHG1 contributes to osteosarcoma progression through sequestration of miR-577 and activation of WNT2B/Wnt/ $\beta$-catenin pathway. Biochem Biophys Res Commun 495: 238-245, 2018.

14. Wang J, Cao L, Wu J and Wang Q: Long non-coding RNA SNHG1 regulates NOB1 expression by sponging miR-326 and promotes tumorigenesis in osteosarcoma. Int J Oncol 52: 77-88, 2018.

15. Lu TX and Rothenberg ME: MicroRNA. J Allergy Clin Immunol 141: 1202-1207, 2018.

16. Fei D, Sui G, Lu Y, Tan L, Dongxu Z and Zhang K: The long non-coding RNA-ROR promotes osteosarcoma progression by targeting miR-206. J Cell Mol Med 23: 1865-1872, 2019.

17. Cheng Z, Shu H, Cui Y, Zhang Q, Zhao B, Pan D, Chao Q and Wang D: MiR-424-5p inhibits proliferation, invasion and promotes apoptosis and predicts good prognosis in glioma by directly targeting BFAR. Pathol Oncol Res 26: 2327-2335, 2020.

18. Wu JB, Yang B, Zhang Y, Feng X, He B, Xie H, Zhou L, Wu J and Zheng S: miR-424-5p represses the metastasis and invasion of intrahepatic cholangiocarcinoma by targeting ARK5. Int J Biol Sci 15: 1591-1599, 2019.

19. Wang K, Zhu G, Bao S and Chen S: Long non-coding RNA LINC00511 mediates the effects of ESR1 on proliferation and invasion of ovarian cancer through miR-424-5p and miR-370-5p. Cancer Manag Res 11: 10807-10819, 2019.

20. Long XH, Mao JH, Peng AF, Zhou Y, Huang SH and Liu ZL: Tumor suppressive microRNA-424 inhibits osteosarcoma cel migration and invasion via targeting fatty acid synthase. Exp Ther Med 5: 1048-1052, 2013.

21. Livak KJ and Schmittgen TD: Analysis of relative gene expression data using real-time quantitative PCR and the 2(-Delta Delta C(T)) method. Methods 25: 402-408, 2001.
22. Huang Y, Liu W, He B, Wang L, Zhang F, Shu H and Sun L: Exosomes derived from bone marrow mesenchymal stem cells promote osteosarcoma development by activating oncogenic autophagy. J Bone Oncol 21: 100280, 2020.

23. Yang $\mathrm{C}$, Wu $\mathrm{K}$, Wang $\mathrm{S}$ and Wei G: Long non-coding RNA XIST promotes osteosarcoma progression by targeting YAP via miR-195-5p. J Cell Biochem 119: 5646-5656, 2018.

24. Zhao J, Zhang C, Gao Z, Wu H, Gu R and Jiang R: Long non-coding RNA ASBEL promotes osteosarcoma cell proliferation, migration, and invasion by regulating microRNA-21. J Cell Biochem 119: 6461-6469, 2018.

25. Xu R, Feng F, Yu X, Liu Z and Lao L: LncRNA SNHG4 promotes tumour growth by sponging miR-224-3p and predicts poor survival and recurrence in human osteosarcoma. Cell Prolif 51: e12515, 2018.

26. Dong P, Xiong Y, Yue J, Hanley SJB and Watari H: miR-34a, miR-424 and miR-513 inhibit MMSET expression to repress endometrial cancer cell invasion and sphere formation. Oncotarget 9: 23253-23263, 2018.

27. Wang J, Wang S, Zhou J and Qian Q: miR-424-5p regulates cell proliferation, migration and invasion by targeting doublecortin-like kinase 1 in basal-like breast cancer. Biomed Pharmacother 102: 147-152, 2018.

28. Fang Y, Liang X, Xu J and Cai X: miR-424 targets AKT3 and PSAT1 and has a tumor-suppressive role in human colorectal cancer. Cancer Manag Res 10: 6537-6547, 2018.

29. Shekhar R, Priyanka P, Kumar P, Ghosh T, Khan MM, Nagarajan P and Saxena S: The microRNAs miR-449a and miR-424 suppress osteosarcoma by targeting cyclin A2 expression. J Biol Chem 294: 4381-4400, 2019.

30. Yamanaka Y, Friess H, Buchler M, Beger HG, Uchida E, Onda M, Kobrin MS and Korc M: Overexpression of acidic and basic fibroblast growth factors in human pancreatic cancer correlates with advanced tumor stage. Cancer Res 53: 5289-5296, 1993.

31. Berger W, Setinek U, Mohr T, Kindas-Mügge I, Vetterlein M, Dekan G, Eckersberger F, Caldas C and Micksche M: Evidence for a role of FGF-2 and FGF receptors in the proliferation of non-small cell lung cancer cells. Int J Cancer 83: 415-423, 1999.

32. Gazzaniga P, Gandini O, Gradilone A, Silvestri I, Giuliani L, Magnanti M, Gallucci M, Saccani G, Frati L and Agliano AM: Detection of basic fibroblast growth factor mRNA in urinary bladder cancer: Correlation with local relapses. Int J Oncol 14: 1123-1127, 1999.

33. Zhang QH, Xu P, Lu YX and Dou HT: Acidic and basic fibroblast growth factor expression levels in cervical cancer and their effects on tumor cell proliferation. Genet Mol Res 15, 2016

34. Cheng Z, Ma R, Tan W and Zhang L: MiR-152 suppresses the proliferation and invasion of NSCLC cells by inhibiting FGF2. Exp Mol Med 46: e112, 2014

35. Sun XH, Geng XL,Zhang J and Zhang C: miRNA-646 suppresses osteosarcoma cell metastasis by downregulating fibroblast growth factor 2 (FGF2). Tumour Biol 36: 2127-2134, 2015. 